\title{
A Unified Bayesian Approach to Multi-Frame Super-Resolution and Single-Image Upsampling in Multi-Sensor Imaging
}

Thomas Köhler ${ }^{12}$

thomas.koehler@fau.de

Johannes Jordan ${ }^{1}$

johannes.jordan@fau.de

Andreas Maier ${ }^{12}$

andreas.maier@fau.de

Joachim Hornegger ${ }^{12}$

joachim.hornegger@fau.de

\author{
${ }^{1}$ Pattern Recognition Lab, \\ Department of Computer Science, \\ Friedrich-Alexander-Universität Erlangen-Nürnberg \\ Erlangen, Germany \\ ${ }^{2}$ Erlangen Graduate School in Advanced Optical Technologies \\ (SAOT) \\ Erlangen, Germany
}

Multi-sensor imaging has become an emerging field of research with a vast number of applications including color and multispectral cameras or 3-D range imaging to capture RGB-D data. One common property of these systems is that they provide multi-channel images that are, unfortunately, often limited in terms of their spatial resolution. However, channel-wise resolution enhancement might not be optimal as it ignores dependencies across the channels. This is obvious if structures are visible in multiple channels. For this reason, tailor-made approaches for single-image upsampling and multi-frame super-resolution have been investigated in different domains. Super-resolution of color images exploits the correlation of color channels as a prior to super-resolve them [2]. In multispectral imaging, super-resolution can be guided by panchromatic data [1]. A similar concept has been established in range imaging, where high-quality color images guide single-image upsampling [3, 5] or multi-frame super-resolution [6] of range images. To avoid the need of reliable guidance data, joint resolution enhancement of all channels has been proposed [4]. However, this formulation does only consider simplified setups with two image channels.

Bayesian Multi-Sensor Model. This work introduces multi-frame superresolution and single-image upsampling that exploit correlations across complementary channels in a unified way. Unlike prior work, this framework is neither limited to a certain setup nor a fixed number of channels and does not require guidance data. Our framework is derived from a Bayesian model to infer a high-resolution multi-channel image $\boldsymbol{x}$ assembled from $n$ channels $x_{1}, \ldots, x_{n}$ from multiple low-resolution multi-channel images $\boldsymbol{y}_{1}, \ldots, \boldsymbol{y}_{K}$. As a key idea, we formulate an image prior according to:

$$
p\left(\boldsymbol{x}_{i} \mid \mathcal{X}_{i}\right) \propto \exp \left\{-\left(\lambda_{i} R_{\text {intra }}\left(\boldsymbol{x}_{i}\right)+\sum_{j=1, j \neq i}^{n} \mu_{i j} R_{\text {inter }}\left(\boldsymbol{x}_{i}, \boldsymbol{x}_{j} ; \boldsymbol{\Phi}_{i j}\right)\right)\right\},
$$

where $\mathcal{X}_{i}=\left\{\boldsymbol{x}_{1}, \ldots, \boldsymbol{x}_{i-1}, \boldsymbol{x}_{i+1}, \ldots, \boldsymbol{x}_{n}\right\}$ and consider two aspects: 1) Each channel $\boldsymbol{x}_{i}$ is described by an intra-channel prior defined by the regularization term $R_{\text {intra }}\left(\boldsymbol{x}_{i}\right)$ with the regularization weight $\lambda_{i} \geq 0$. 2) Dependencies across channels $\boldsymbol{x}_{i}$ and $\boldsymbol{x}_{j}$ are modeled by an inter-channel prior $R_{\text {inter }}\left(\boldsymbol{x}_{i}, \boldsymbol{x}_{j} ; \boldsymbol{\Phi}_{i j}\right)$ with pair-wise weights $\mu_{i j} \geq 0$ and hyperparameters $\boldsymbol{\Phi}_{i j}$. Locally Linear Regression. The inter-channel prior is defined by locally linear regression (LLR) that explains dependencies across channels by:

$$
R_{\text {inter }}\left(\boldsymbol{x}_{i}, \boldsymbol{x}_{j} ; \boldsymbol{\Phi}_{i j}\right)=\left\|\kappa\left(\boldsymbol{x}_{i}, \boldsymbol{x}_{j}\right) \odot\left(\boldsymbol{A}_{i j} \boldsymbol{x}_{i}+\boldsymbol{b}_{i j}-\boldsymbol{x}_{j}\right)\right\|_{2}^{2},
$$

where the hyperparameters $\boldsymbol{\Phi}_{i j}$ are given by filter coefficients $\boldsymbol{A}_{i j}$ and $\boldsymbol{b}_{i j}$ for each channel pair $\left(\boldsymbol{x}_{i}, \boldsymbol{x}_{j}\right)$ and associated confidence weights $\kappa\left(\boldsymbol{x}_{i}, \boldsymbol{x}_{j}\right)$.
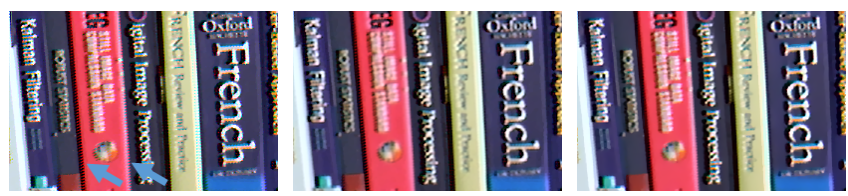

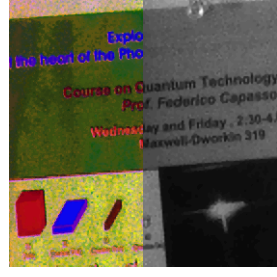

(a) Channel-wise

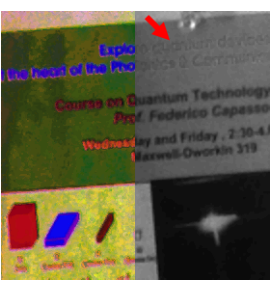

(b) Multi-channel $[2$

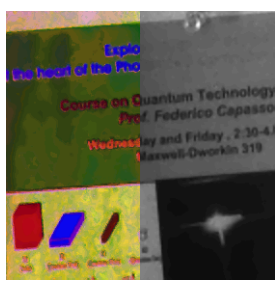

(c) Multi-channel proposed
Figure 1: Comparison of multi-frame color super-resolution (top row) and single-image upsampling of multispectral data (bottom row).
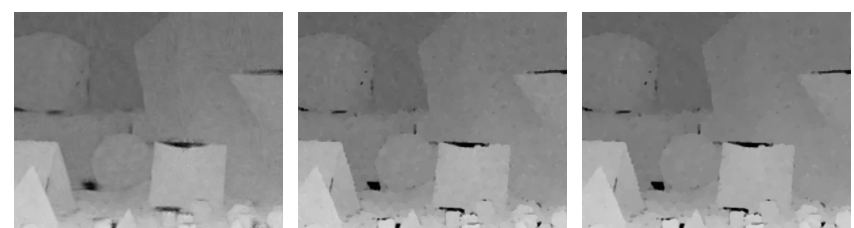

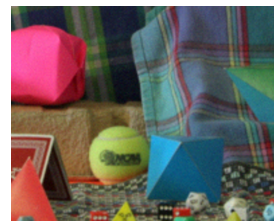

(a) Guided upsampling [5]

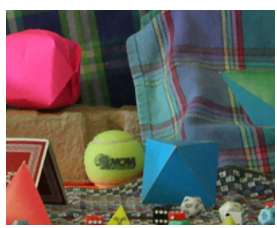

(b) Channel-wise

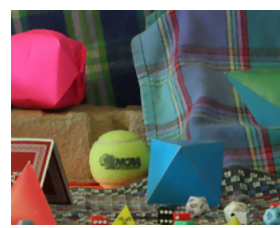

(c) Multi-channel proposed
Figure 2: Single-image upsampling of RGB-D data.

This prior is spatially adaptive and tolerates outliers due to image regions that violate the LLR assumption. The inference of the hyperparameters $\boldsymbol{\Phi}_{i j}$ that are treated as latent variables as well as the multi-channel image $\boldsymbol{x}$ are formulated as maximum a-posteriori (MAP) estimation. For this purpose, our paper presents an efficient alternating minimization scheme.

Applications. We evaluated our unified approach for color-, multispectral and range imaging. In color and multispectral imaging, we compared the proposed inter-channel prior to conventional channel-wise processing as well as the color channel regularization of Farsiu et al. [2], see Fig.1] Our multi-channel method got rid of color artifacts present in channel-wise super-resolution, e. g. jagged edges highlighted in Fig.1(top). Unlike [2] that erroneously copied structure from other, original channels to the reconstructed channel, this issue was avoided by our adaptive confidence weighting as highlighted for multispectral data in Fig.1 (bottom). In range imaging, our method was evaluated for single-image upsampling, where we compared channel-wise and guided upsampling [5], see Fig. 2 Unlike [5], our multi-channel approach does not rely on high-quality color images as guidance. In particular, it achieved better reconstructions of surfaces and edges in range images compared to channel-wise and guided upsampling.

[1] M. Aguena and N. Mascarenhas. Multispectral image data fusion using POCS and super-resolution. Computer Vision and Image Understanding, 102(2):178-187, 2006.

[2] S. Farsiu, M. Elad, and P. Milanfar. Multiframe demosaicing and superresolution of color images. IEEE Transactions on Image Processing, 15(1):141-159, 2006.

[3] D. Ferstl, C. Reinbacher, R. Ranftl, M. Ruether, and H. Bischof. Image Guided Depth Upsampling Using Anisotropic Total Generalized Variation. In Proc. IEEE Conference on Computer Vision and Pattern Recognition, pages 993-1000, 2013.

[4] F. C. Ghesu, T. Köhler, S. Haase, and J. Hornegger. Guided image super-resolution: A new technique for photogeometric superresolution in hybrid 3-d range imaging. In Pattern Recognition, pages 227-238. Springer, 2014.

[5] K. He, J. Sun, and X. Tang. Guided image filtering. IEEE Transactions on Pattern Analysis and Machine Intelligence, 35(6):1397-409, 2013.

[6] T. Köhler, S. Haase, S. Bauer, J. Wasza, T. Kilgus, L. Maier-Hein, C. Stock, J. Hornegger, and H. Feußner. Multi-sensor super-resolution for hybrid range imaging with application to 3-d endoscopy and open surgery. Medical Image Analysis, 24(1):220-234, 2015. 